\title{
Production, Physico-Chemical Characterization and Biodistribution Studies of Lipid Nanoparticles
}

Elisabetta Esposito', Helga Eveline De Vries², Susanne M.A. van der Pol ${ }^{2}$, Federico Boschi ${ }^{3}$, Laura Calderan ${ }^{4}$, Silvia Mannucci ${ }^{4}$, Markus Drechsler ${ }^{5}$, Catia Contado ${ }^{6}$, Rita Cortesi ${ }^{1}$ and Claudio Nastruzzi ${ }^{1 *}$

${ }^{1}$ Department of Life Sciences and Biotechnology, University of Ferrara, 44121 Ferrara, Italy

${ }^{2}$ Department of Molecular Cell Biology and Immunology, Neuroscience Campus Amsterdam, VU University Medical Center, Amsterdam, The Netherlands

${ }^{3}$ Department of Computer Sciences, University of Verona, 37134 Verona, Italy

${ }^{4}$ Department of Neurological and Movement Sciences, University of Verona, 37134 Verona, Italy

${ }^{5}$ BIMF/Soft Matter Electron microscopy, University of Bayreuth, Germany

${ }^{6}$ Department of Chemistry, University of Ferrara, 44121 Ferrara, Italy

\begin{abstract}
This study describes the preparation, characterization, in vitro uptake and in vivo biodistribution in mice of solid lipid nanoparticles and nanostructured lipid carriers.

The effect of nanoparticle lipid matrix, presence of fluorescent and functionalization by polysorbate 80 on dimensional distribution and morphology have been studied by sedimentation field flow fractionation, photon correlation spectroscopy and cryogenic transmission electron microscopy. The complementary use of different techniques demonstrated that lipid matrix composition, presence of fluorescent dye and polysorbate 80 functionalization have little effect on nanoparticle morphology and size distribution.
\end{abstract}

Uptake of fluorescent nanoparticles was determined in vitro by human brain endothelial cells, showing that nanoparticles treated by polysorbate 80 displayed lower uptake values with respect to the corresponding control nanoparticles.

Biodistribution of solid lipid nanoparticles treated by polysorbate 80 was evaluated by fluorescent luminescent imaging after intraperitoneal administration in mice. The in vivo images indicate that nanoparticles were able to reach the brain, even if they prevalently accumulated in liver and spleen.

Keywords: Solid lipid nanoparticles; Nanostructured lipid carriers; Cryogenic transmission electron microscopy; Sedimentation field flow fractionation; Photon correlation spectroscopy; In vitro uptake; In vivo biodistribution

Abbreviations: SLN: Solid Lipid Nanoparticles; NLC: Nanostructured Lipid Carriers; : P80: Polysorbate 80; SLN-P80: Solid Lipid Nanoparticles Treated with Polysorbate 80; NLC-P80: Nanostructured Lipid Carriers Treated with Polysorbate 80; SLNCG: Solid Lipid Nanoparticles containing Cardiogreen; SLN-P80CG: Solid Lipid Nanoparticles containing Cardiogreen and Treated with Polysorbate 80; SLN-RH: Solid Lipid Nanoparticles containing Rhodamine; NLC-RH: Nanostructured Lipid Carriers containing Rhodamine; SLN-P80-RH: Solid Lipid Nanoparticles containing Rhodamine and Treated with Polysorbate 80; NLC-P80-RH: Nanostructured Lipid Carriers containing Rhodamine and Treated with Polysorbate 80; I.P: Intraperitoneal; Cryo-TEM: Cryogenic Transmission Electron Microscopy; SdFFF: Sedimentation Field Flow Fractionation; PCS: Photon Correlation Spectroscopy; BBB: Blood Brain Barrier; EE: Encapsulation Efficiency; LC: Loading Capacity

\section{Introduction}

In recent years lipid nanoparticles have been proposed in a number of pharmaceutical research studies as drug delivery formulations, due to their versatility and advantages $[1,2]$. For instance, the lipid based excipients constituting their solid matrix: (i) allow the solubilization of lipophilic molecules in a biocompatible and biodegradable environment with well-established safety profiles, (ii) improve the stability of labile molecules and (iii) ensure controlled release profile $[2,3]$.

The chance of producing lipid nanoparticles by simple emulsification of the molten lipids and subsequent recrystallization, eludes the use of potentially toxic organic solvents $[3,4]$. Moreover lipid nanoparticles can be administered by different routes and targeted to specific organs, such as the brain. At this regard, many investigations have been published, describing the use of lipid nanoparticles as delivery system able to overcome the blood brain barrier (BBB) [5-7].

Depending on the nature of the lipids constituting nanoparticle matrix, solid lipid nanoparticles (SLN) or nanostructured lipid carriers (NLC) can be distinguished.

The former are composed of lipids in solid state at room and body temperatures, and the latter are constituted of mixtures of solid and liquid lipids [8-10].

Notwithstanding the potential suitability of lipid nanoparticle as drug delivery system, it is to be considered that intravenously injected SLN and NLC are rapidly cleared from the systemic circulation by opsonisation $[7,11,12]$. In order to overcome this drawback, in recent years, research has been conducted to create "stealth" nanoparticles characterized by longer circulation times, avoiding preferential uptake by the Reticulo Endothelial System (RES) and promoting in this way the possibility of reaching specific organs, like the brain $[13,14]$. At this

*Corresponding author: Claudio Nastruzzi, Department of Life Sciences and Biotechnology, Via Fossato di Mortara, 19 l-44121 Ferrara, Italy, Tel: 39/0532/455348; Fax: 39/0532/455953; E-mail: nas@unife.it

Received November 11, 2014; Accepted December 09, 2014; Published January 01,2015

Citation: Esposito E, Vries HED, Pol SMA, Boschi F, Calderan L, et al. (2015) Production, Physico-Chemical Characterization and Biodistribution Studies of Lipid Nanoparticles. J Nanomed Nanotechnol 6: 256. doi: 10.4172/2157-7439.1000256

Copyright: ( $) 2015$ Esposito E, et al. This is an open-access article distributed under the terms of the Creative Commons Attribution License, which permits unrestricted use, distribution, and reproduction in any medium, provided the original author and source are credited. 
Citation: Esposito E, Vries HED, Pol SMA, Boschi F, Calderan L, et al. (2015) Production, Physico-Chemical Characterization and Biodistribution Studies of Lipid Nanoparticles. J Nanomed Nanotechnol 6: 256. doi: 10.4172/2157-7439.1000256

Page 2 of 9

regard different approaches have been proposed, based on nanoparticle surface modification by Pluronic F-68, poly(ethylene glycol) or polysorbate 80 (P80) [15-17].

In order to draw lipid nanoparticle as drug delivery system, it is to be considered the relevance of particle characterization for quality control, stability evaluation and biological fate. Physical properties such as morphology, size and size distribution of nanoparticles heavily affect their biological activity, biodistribution and toxicity $[18,19]$. Meanwhile, since the obtained size distribution strongly depends on the employed method, the combination of complementary measurement techniques is recommended to overcome the impairment of the single ones [20].

Fluorescence molecular tomography can be employed for evaluating nanoparticle biodistribution, enabling a non-invasive detection, visualization and quantification of fluorescence distributed throughout the body of living animals [21,22].

The goal of this study was the design and production of fluorescent lipid nanoparticles suitable for in vitro uptake study by human brain endothelial cells and for biodistribution imaging, focusing on the influence of (a) nanoparticle lipid matrix, (b) presence of fluorescent and (c) presence of P80 on nanoparticle physico-chemical characteristics.

In particular after nanoparticle production and characterization, their uptake was studied in vitro on hCMEC/D3 cell line. Moreover a biodistribution study was performed on mice by in vivo fluorescent luminescent imaging after intraperitoneal (i.p.) administration of fluorescent SLN.

\section{Materials and Methods}

\section{Materials}

The copolymer poly (ethylene oxide) (a) -poly (propylene oxide) (b) $(a=80, b=27)$ (poloxamer 188) was a gift of BASF ChemTrade $\mathrm{GmbH}$ (Burgbernheim, Germany). Tristearin, stearic triglyceride (tristearin) and polysorbate 80 (P80) were provided by Fluka (BuchsSwitzerland). Miglyol 812, caprylic/capric triglycerides (miglyol) was a gift of Cremer Oleo Division (Witten, Germany). 5(6)-Carboxy-X- rhodamine (rhodamine) and indocyanine green (cardiogreen) were from Sigma-Aldrich (Milano, Italy).

\section{Production of lipid nanoparticles}

SLN and NLC were prepared by melt and ultrasonication, following a previously reported method with minor modifications [17]. The lipid phase (5\% with respect to the whole weight of the dispersion) was alternatively constituted of pure tristearin, in the case of SLN, or the mixture tristearin/miglyol 2:1 w/w, in the case of NLC. Briefly, an aqueous poloxamer 188 solution $(2.5 \% \mathrm{w} / \mathrm{w})$ heated at $80^{\circ} \mathrm{C}$ was added to the molten lipid phase, afterwards the mixture was emulsified at $15000 \mathrm{rpm}, 80^{\circ} \mathrm{C}$ for $1 \mathrm{~min}$, subjected to ultrasonication at $6.75 \mathrm{kHz}$ for $15 \mathrm{~min}$ and then let cooling at $25^{\circ} \mathrm{C}$. SLN and NLC dispersions were stored at room temperature. In some cases, P80 $(16.6 \% \mathrm{w} / \mathrm{w}$ with respect to the lipid weight) was added to the dispersion after the ultrasonication step, during cooling $\left(\right.$ at $\left.40^{\circ} \mathrm{C}\right)$ and left under stirring $(250 \mathrm{rpm})$ for $30 \mathrm{~min}$. Table 1 reports the abbreviations used throughout the text to indicate the different nanoparticles.

\section{Production of fluorescent lipid nanoparticles}

In order to produce fluorescent nanoparticles for in vitro uptake and in vivo biodistribution studies, rhodamine and cardiogreen $(0.4 \%$ $\mathrm{w} / \mathrm{w}$ with respect to the weight of the lipids) were respectively employed. Two alternative fluorescent dyes have been employed because of technical and methodological reasons. Indeed the fluorimeters employed for in vitro and in vivo studies require different wavelengths for detection of the fluorophore. For in vitro studies the fluorimeter needs excitation wavelength $560 \mathrm{~nm}$ and emission $590 \mathrm{~nm}$, while the IVIS Spectrum used in fluorescent modality for in vivo experiments has excitation filter $740 \mathrm{~nm}$ and emission filter $800 \mathrm{~nm}$. Accordingly, rhodamine was selected for in vitro experiment while cardiogreen was chosen for in vivo studies, having excitation wavelengths of 570 and 750 $\mathrm{nm}$ respectively. The fluorescent dyes were added to the molten lipid phase and mixed before adding to the aqueous phase. The purification of fluorescent particle from free fluorescent dyes and the determination of fluorescence content of nanoparticles were determined as follows. Three hundred microliter samples of nanoparticle were loaded in a centrifugal filter (Microcon unit YM-10 membrane, NMWCO $10 \mathrm{kDa}$,

\begin{tabular}{|c|c|c|c|c|c|c|}
\hline \multirow{3}{*}{$\begin{array}{l}\text { Identification } \\
\text { code }\end{array}$} & \multicolumn{6}{|c|}{ Nanoparticle formulation constituents } \\
\hline & \multicolumn{4}{|c|}{ Disperse nanoparticle lipid phase ${ }^{a}$} & \multicolumn{2}{|c|}{ Dispersing aqueous phase ${ }^{b}$} \\
\hline & Tristearin & Mygliol & Cardiogreen & Rhodamine & Poloxamer 188 & Polysorbate 80 \\
\hline \#SLN & 100.0 & n.p. & n.p. & n.p. & 2.5 & n.p. \\
\hline \#SLN-P80 & 100.0 & n.p. & n.p. & n.p. & 2.5 & 1.0 \\
\hline \#NLC & 66.6 & 33.3 & n.p. & n.p. & 2.5 & n.p. \\
\hline \#NLC-P80 & 66.6 & 33.3 & n.p. & n.p. & 2.5 & 1.0 \\
\hline \#SLN-RH & 99.6 & n.p. & n.p. & 0.4 & 2.5 & n.p. \\
\hline \#SLN-P80-RO & 99.6 & n.p. & n.p. & 0.4 & 2.5 & 1.0 \\
\hline \#NLC-RH & 66.6 & 33.3 & n.p. & 0.4 & 2.5 & n.p. \\
\hline \#NLC-P80-RH & 66.6 & 33.3 & n.p. & 0.4 & 2.5 & 1.0 \\
\hline \#SLN-CG & 99.6 & n.p. & 0.4 & n.p. & 2.5 & n.p. \\
\hline \#SLN-P80-CG & 99.6 & n.p. & 0.4 & n.p. & 2.5 & 1.0 \\
\hline
\end{tabular}

n.p.: not present;

athe values are expressed as weight percentage with respect to the lipid phase constituting the nanoparticle matrix;

bthe values are expressed as weight percentage with respect to the dispersing aqueous phase; in all the batches the total weight of the disperse lipid phase is $5 \%$ w/w with respect to the whole weight of the dispersion

Table 1: Identification codes and chemical composition of the nanoparticles used in the present study. 
Sigma Aldrich, St Louis, MO, USA) and centrifuged (Spectrafuge ${ }^{\mathrm{max}} 24 \mathrm{D}$ Digital Microcentrifuge, Woodbridge NJ, USA) at 8,000 rpm for 60 min. The fluorescence present in the eluate from the centrifugation was thereafter determined by fluorescence spectroscopy, taking as reference the previously made calibration curve. The encapsulation efficiency (EE) and loading capacity (LC) were determined by fluorescent spectroscopy. The encapsulation parameters were determined as follows:

$$
\begin{aligned}
& \mathrm{EE}=\mathrm{T}_{\text {fluo }}-\mathrm{S}_{\text {fluo }} / \mathrm{T}_{\text {fluo }} \times 100 \\
& \mathrm{LC}=\mathrm{T}_{\text {fluo }}-\mathrm{S}_{\text {fluo }} / \mathrm{T}_{\text {lipid }} \times 100
\end{aligned}
$$

where $\mathrm{T}_{\text {fluo }}$ stands for the total amount of fluorescent dye added to the nanoparticles; $\mathrm{S}_{\text {fluo }}$ for the amount of fluorescent dye measured in the eluate, and $\mathrm{T}_{\text {lipid }}$ for the total weight of lipid in the formulation. The association of fluorescent dye to nanoparticle was verified by thin-layer chromatography (TLC) utilizing TLC silica gel 60 F254 (Merck KGaA, Darmstadt, Germany) with an imaging plate (BAS-SR2040, FujiFilm, Tokyo, Japan) and using a fluorescence imaging system (photodynamic eye (PDE); Hamamatsu Photonics, Hamamatsu, Japan). TLC was performed applying on the TLC plate $5 \mu \mathrm{l}$ of the fluorescent dye in nanoparticle or as solution, using as eluents mixtures of ethanol/water $85: 15 \mathrm{v} / \mathrm{v}$ or $96: 4 \mathrm{v} / \mathrm{v}$, for rhodamine or cardiogreen, respectively. The fluorescent spots were quantified $25 \mathrm{~min}$ later. TLC plates were put on the imaging plate and then scanned using a phosphor imager FLA2000 (FujiFilm, Tokyo, Japan). Fluorescence levels were visualized from the display.

\section{Characterization of lipid nanoparticles}

The macroscopic aspect of lipid nanoparticles was evaluated under visual inspection to detect possible phase separation, aggregation and sedimentation phenomena. The visual inspection was also performed after serial dilution with poloxamer 188 solution $(2.5 \% \mathrm{w} / \mathrm{w})$, resulting in $56,28,14,7$ and $3.5 \mathrm{mM}$ final lipid concentration.

Sedimentation Field Flow Fractionation (SdFFF) analysis: A SdFFF system (Model S101, FFFractionation, Inc., Salt Lake City, UT, USA), described elsewhere, was employed to determine the size distribution of particles by converting the fractograms, i.e. the graphical results, assuming that the particle density is known [23]. The mobile phase was demineralized water pumped at $2.0 \mathrm{ml} / \mathrm{min}$ and monitored in each run. Fifty microliter samples were injected as they were through a $50 \mu$ l Rheodyne loop valve. After the SdFFF analyses, the fractions were automatically collected by a Model 2110 fraction collector (Bio Rad laboratories, UK) with a collecting time of $90 \mathrm{sec}$. The volume of each fraction was $3 \mathrm{ml}$.

Photon Correlation Spectroscopy (PCS) analysis: Submicron particle size analysis was performed using a Zetasizer 3000 PCS (Malvern Instr., Malvern, England) equipped with a $5 \mathrm{~mW}$ helium neon laser with a wavelength output of $633 \mathrm{~nm}$. Glassware was cleaned of dust by washing with detergent and rinsing twice with water for injections. Measurements were made at $25^{\circ} \mathrm{C}$ at an angle of $90^{\circ}$. Data were interpreted using the "CONTIN" method [24].

Cryogenic transmission electron microscopy (cryo-TEM) analysis: Samples were vitrified as previously described [13]. The vitrified specimen was transferred to a Zeiss EM922Omega transmission electron microscope for imaging using a cryoholder (CT3500, Gatan). The temperature of the sample was kept below $-175^{\circ} \mathrm{C}$ throughout the examination. Specimens were examined with doses of about 1000-2000 $\mathrm{e} / \mathrm{nm}^{2}$ at $200 \mathrm{kV}$. Images were recorded digitally by a CCD camera (Ultrascan 1000, Gatan) using an image processing system (GMS 1.9 software, Gatan). In addition size distribution of nanoparticles was performed by measuring 1000 nanoparticles for each cryo-TEM image by the digital analyzer Image $1.48 \mathrm{v}$.

\section{Stability studies}

Stability studies were conducted on nanoparticles stored for 180 days at room temperature. PCS size distribution was analyzed in triplicate weekly, up to 60 days and monthly, up to 180 days from nanoparticle production. Morphology of nanoparticles was checked by cryo-TEM observation after 90 days from production.

\section{In vitro uptake experiments}

For the in vitro uptake experiments, the human brain endothelial cell line hCMEC/D3 was employed. These cells derived from a primary culture of human adult brain endothelial cells immortalized via lentiviral transduction of human telomerase reverse transcriptase (hTERT) and simian virus 40 (SV40) large $\mathrm{T}$ antigen [25]. The immortalized human brain endothelial cell line hCMEC/D3, which displays key features of the blood-brain barrier (BBB), was cultured as described previously [25,26]. Nanoparticle uptake by human brain endothelial cell monolayers was analyzed in hCMEC/D3 cells that were seeded at confluence onto collagen-coated 96-wells black plates (Corning Incorporated). Cells were incubated with rhodamine containing nanoparticles, namely SLN-RH, NLC-RH, SLN-P80-RH or NLC-P80-RH, for 24 hours in increasing concentration: $3.125 \mathrm{mg} / \mathrm{ml}$, $1.56 \mathrm{mg} / \mathrm{ml}, 0.78 \mathrm{mg} / \mathrm{ml}, 0.39 \mathrm{mg} / \mathrm{ml}$ in culture medium. After 24 hours the cells were washed with PBS and uptake was determined using the

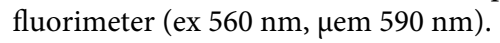

\section{Biodistribution studies}

Male, athymic mice $(\mathrm{n}=8)$ (Harlan Laboratories, Italy), about 4-5 weeks old and $25 \mathrm{~g}$ in weight, were housed in a temperature- and humidity- controlled environment, having free access to mouse chow and tap water. Animals were handled accordingly with the regulations of the Italian Ministry of Health and to the European Communities Council (86/609/EEC) directives. Mice were divided into two groups, the first group $(n=4)$ was administered with an i.p. injection with 500 $\mu \mathrm{l}$ of SLN-P80-CG, the second group $(\mathrm{n}=4)$ was administered by an i.p. injection with $500 \mu \mathrm{l}$ of SLN-P80. Mice were imaged 4 hours after lipid nanoparticle injection. Optical images were acquired with IVIS Spectrum (Perkin Elmer) in fluorescent modality with excitation filter $740 \mathrm{~nm}$ and emission filter $800 \mathrm{~nm}$. Other parameters were: exposure time $1 \mathrm{~s}$, binning $\mathrm{B}=4, \mathrm{f} / \mathrm{stop}=2$. After the last acquisition, mice were sacrificed through an anaesthetic overdose and they were perfused with solutions of phosphate buffer saline and paraformaldehyde. After perfusion liver, brain, lungs, kidney and spleen were extracted and imaged with the same parameters of the in vivo acquisitions.

\section{Results}

\section{Preparation of lipid nanoparticles}

Production of SLN and NLC was performed by a two-step protocol based on the emulsification of the molten lipid phase in an aqueous phase containing poloxamer 188 under high speed stirring at 15000 $\mathrm{rpm}$, followed by treatment with probe ultrasound. Table 1 reports the composition of nanoparticles and the batch identification codes.

After preparation, the nanoparticle dispersions appeared milky and free of aggregates and sediment; in the case of fluorescent nanoparticle, the presence of the fluorescent dyes cardiogreen and rhodamine resulted in milky dispersions respectively green or pink coloured 
Citation: Esposito E, Vries HED, Pol SMA, Boschi F, Calderan L, et al. (2015) Production, Physico-Chemical Characterization and Biodistribution Studies of Lipid Nanoparticles. J Nanomed Nanotechnol 6: 256. doi: 10.4172/2157-7439.1000256

Page 4 of 9

(Figure 1). Serial dilutions of the nanoparticle dispersion resulted in a progressive decrease of the scattering intensity and therefore in the milkiness of the formulations.

The determination of EE and LC was performed after centrifugation of the dispersions, indicating that both dyes were almost quantitatively encapsulated within the lipid matrix of the nanoparticles, as proved by the very high EE, reported in Table 2. Conversely, the LC values were quite low since the tracking molecules rhodamine and cardiogreen were employed as tools to respectively obtain in vitro and in vivo data about nanoparticle uptake and biodistribution. Therefore only the amounts of trackers needed for the specific determinations were included. Nevertheless, previous studies from our research group demonstrated that different drugs could be entrapped in lipid nanoparticles in amounts higher enough to exert a therapeutic effect $[13,17,27]$.

The stability of the association between fluorescent dye and nanoparticles was determined by TLC experiment that confirmed the absence of free fluorescent spots. These experiments strongly indicated that the dyes, due to their lipophilic character, were strongly bond to the nanoparticle lipid phase.

\section{Functionalization of nanoparticles by polysorbate 80}

SLN and NLC have been largely proposed as drug formulations, nonetheless their suitability to deliver drug to the brain has been not yet completely achieved. The difficulties arose mainly in reason of the nanoparticle adsorption by opsonins and their subsequent clearance from the body by phagocytic cells. At this regard researchers have been trying to design long circulating nanocarriers by introducing the stealth nanoparticle approach. This goal was achieved by modifying the nanoparticle surface in a way to facilitate the evasion of clearance by the body and prolonging the circulation time for extended periods $[28,29]$.

Following this general approach, in the present study the

$\mathbf{A}$

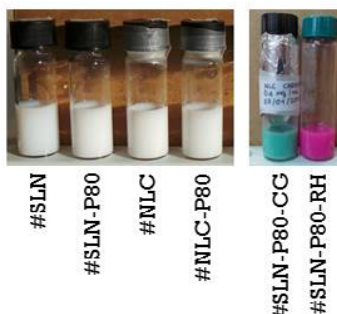

B

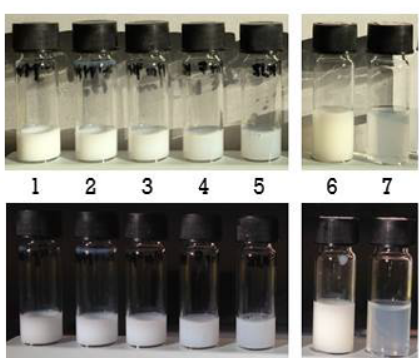

Figure 1: A: Macroscopic analysis of the investigated empty and fluorescent dye containing nanoparticle formulations. B: Bright and dark field photographic analysis of the effect of serial dilution (with poloxamer $188,2.5 \% \mathrm{w} / \mathrm{w}$ ) of SLN-P80 on the general aspect (i.e milkiness) of the formulation. The total lipid content of the shown formulations is 56 (1), 28 (2), 14 (3), 7 (4) 3.5 (5) mM, for comparison images of liposomal suspensions constituted of phospahtidylcholine/cholesterol 36/3.6 (6) and 9/0.9 (7) $\mathrm{mM}$ are also reported. For nanoparticle identification codes, composition and preparation procedure, please refer to Table 1 and experimental section.

\begin{tabular}{|c|c|c|}
\hline \multirow{2}{*}{$\begin{array}{l}\text { Identification } \\
\text { code }\end{array}$} & \multicolumn{2}{|c|}{ Fluorescent encapsulation parameters } \\
\hline & $\begin{array}{c}\text { Encapsulation efficiency }{ }^{a} \\
(\%) \pm S D\end{array}$ & $\begin{array}{c}\text { Loading Capacity }(\%) \\
\pm \text { SD }\end{array}$ \\
\hline \#SLN-RH & $99.0 \pm 0.3$ & $0.4 \pm 0.1$ \\
\hline \#SLN-P80-RH & $95.0 \pm 0.1$ & $0.3 \pm 0.1$ \\
\hline \#NLC-RH & $98.1 \pm 0.2$ & $0.4 \pm 0.1$ \\
\hline \#NLC-P80-RH & $97.0 \pm 0.3$ & $0.3 \pm 0.1$ \\
\hline \#SLN-CG & $95.2 \pm 0.2$ & $0.3 \pm 0.1$ \\
\hline \#SLN-P80-CG & $96.1 \pm 0.3$ & $0.3 \pm 0.1$ \\
\hline
\end{tabular}

aPercentage $(\mathrm{w} / \mathrm{w})$ of fluorescent in the whole dispersion with respect to the tota

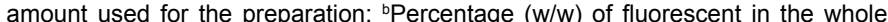
dispersion with respect to the amount of lipid used for the preparation. Data are means of 4 independent determinations.

Table 2: Fluorescent encapsulation parameters in nanoparticles.

\begin{tabular}{|c|c|c|c|c|c|c|}
\hline \multirow{3}{*}{\begin{tabular}{|c|} 
Identification \\
code
\end{tabular}} & \multicolumn{6}{|c|}{ Analytical method } \\
\hline & \multicolumn{2}{|c|}{ SdFFF* } & \multicolumn{2}{|c|}{$\mathrm{PCS}^{\circ}$} & \multicolumn{2}{|c|}{ Cryo-TEM } \\
\hline & $\begin{array}{l}\text { Mean } \\
\text { diameter } \\
(\mathrm{nm})\end{array}$ & s.d. & $\begin{array}{c}\text { Mean } \\
\text { diameter } \S \\
(\mathrm{nm})\end{array}$ & s.d. & $\begin{array}{c}\text { Mean } \\
\text { diameter } \\
(\mathrm{nm})\end{array}$ & s.d. \\
\hline \#SLN & 44.6 & 3 & 166.1 & 5 & 113.7 & 9 \\
\hline \#SLN-P80 & 32.0 & 2 & 169.3 & 4 & 112.6 & 2 \\
\hline \#NLC & 60.6 & 5 & 152.5 & 4 & 119.0 & 8 \\
\hline \#NLC-P80 & 59.6 & 4 & 162.2 & 6 & 78.7 & 6 \\
\hline \#SLN-CG & 49.5 & 3 & 172.6 & 7 & 97.2 & 7 \\
\hline \#SLN-P80-CG & 32.9 & 2 & 183.4 & 11 & 88.7 & 7 \\
\hline
\end{tabular}

*Sedimentation Field Flow Fractionation;

'Photon Correlation Spectroscopy; "Cryogenic Transmission Electron Microscopy; §expressed as Z-Average;

s.d.: standard deviation; data are the mean of 5 measurements

Table 3: Mean diameters of the nanoparticles used in the present study, as determined by SdFFF, PCS and Cryo-TEM.

nanoparticles have been treated post-production, with the non-toxic surfactant P80, approved by the Food and Drug Administration also for intravenous administration [30]. Notably, the addition of P80 to the nanoparticle was performed at $40^{\circ} \mathrm{C}$, this procedure allowed the formation of a P80 coating on the nanoparticles through the penetration of the hydrophobic chains of the surfactant within the lipid matrix. On the contrary, the polyoxyethylene chains of the surfactant, in reason of their hydrophilic character, protrude into the water phase, creating a hydrophilic cloud at the particle-water interface. The addition of P80 did not change the macroscopic aspect, with respect to untreated SLN or NLC, as shown in Figure 1.

\section{Characterization of nanoparticle dispersions}

It is well known that size distribution and shape are important parameters influencing physico-chemical stability and in vivo fate of nanoparticles [19].

Since analytical methods for dimensional and morphology determination are based on different physical principles and can lead to misleading interpretations, in the present investigation three techniques were employed in order to obtain reliable results.

In particular, Table 3 reports the mean diameters measured by SdFFF, PCS and cryo-TEM analyses. In addition, Figure 1 reports the nanoparticle size distributions obtained by SdFFF. The cumulative frequency distributions of lipid nanoparticles produced in the absence (A) and in the presence of P80 (B), or nanoparticles treated by P80 containing the fluorescent dye cardiogreen $(\mathrm{C})$ are compared. 
The lipid matrix composition did not significantly affect nanoparticle mean diameter and size distribution (Figure 1A), SLN and NLC presented indeed very similar values of both size and size distribution. Whereas SLN treated by P80 showed a reduction of the mean diameter and a narrowing of the size distribution with respect to the NLC counterpart (Figure 1B).

In agreement to the macroscopic observation, SdFFF analysis confirmed that the presence of cardiogreen (Figure 1C) had very little effect on nanoparticle dimensions; the addition of polysorbate 80 to fluorescent SLN lead to a slight decrease in mean diameter (Table 3). Rhodamine containing nanoparticles displayed dimensions perfectly superposable to those of cardiogreen nanoparticles (data not shown).

Figure 2 reports the Z-Average (A) and dispersity (B) values determined by PCS technique. The lipid composition and the presence of both fluorescent and P80 slightly affected nanoparticle dimensions, with mean diameters comprised between 166 and $183 \mathrm{~nm}$; the dispersity was always $<0.2$, indicating a narrow size distribution.

Nanoparticle dimensions were also determined by microscopic analysis. Cryo-TEM images were studied by computer aided image analysis allowing the determination of mean diameters. The results, reported in Table 3, confirmed that nanoparticle were only marginally
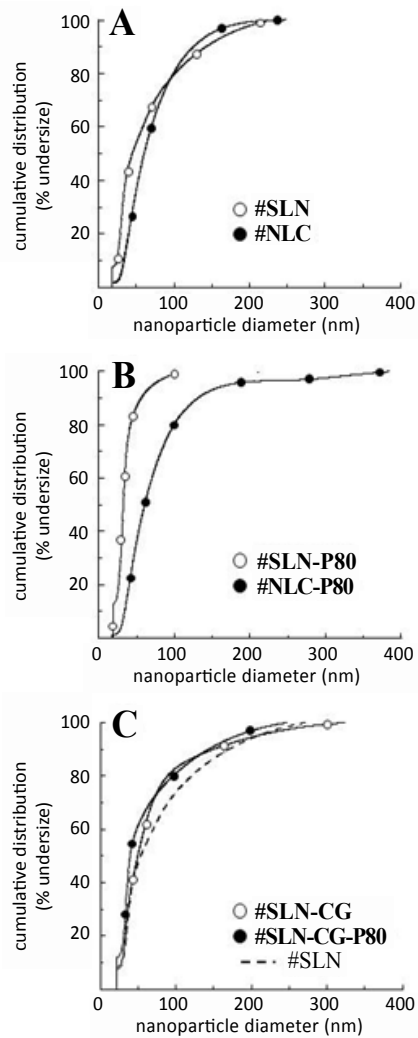

Figure 2: Effect of lipid nanoparticle constituents, presence of fluorescent dye and production parameters on particle dimensions, as determined by sedimentation field flow fractionation analysis.

Graphs show the cumulative size distribution analysis (by number) of solid lipid nanoparticles (open circles) and nanostructured lipid carriers (closed circles) (A), lipid nanoparticles treated by polysorbate 80 (B) or lipid nanoparticles treated by polysorbate 80 containing the fluorescent dye cardiogreen (C). For nanoparticle identification codes, composition and preparation procedure please refer to Table 1 and experimental section.
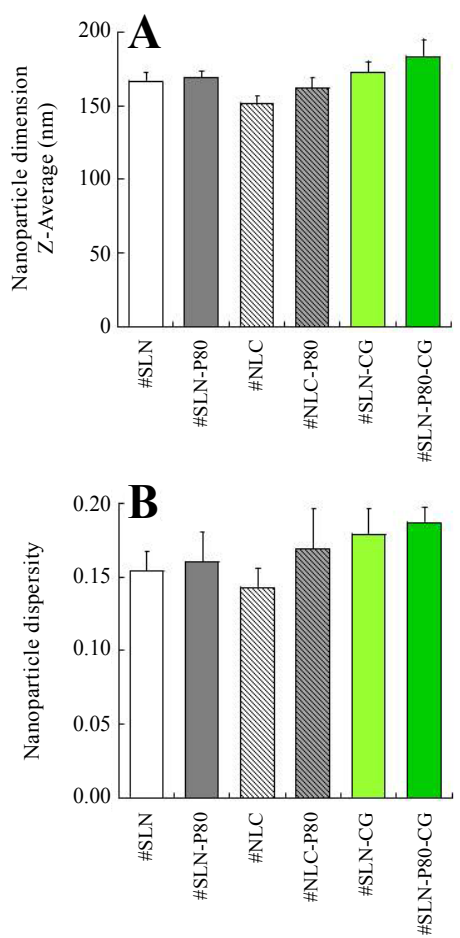

Figure 3: Effect of lipid nanoparticle constituents, presence of fluorescent dye and production parameters on particle dimensions, as determined by photon correlation spectroscopy analysis.

Graphs show the Z-average $(A)$ and dispersity $(B)( \pm S D, n=5)$ of lipid nanoparticles constituted of solid lipids (plain bars), a mixture of solid and liquid lipids (striped bars) or solid lipids plus the fluorescent dye cardiogreen (green bars).After production, lipid nanoparticle were incubated at $40^{\circ} \mathrm{C}$, for $30 \mathrm{~min}$ in the absence (light bars) or in the presence of polysorbate 80 (dark bars). For nanoparticle identification codes, composition and preparation procedure please refer to Table 1 and experimental section.

affected by lipid composition, presence of dye or P80 treatment.

As general comment on size determinations, nanoparticle mean diameters were in decreasing order in the following sequence: PCS $>$ cryo-TEM $>$ SdFFF.

At this regard, the different principles of the three techniques and their limits should be considered. SdFFF allows to separate particles as a function of their specific mass, indeed by knowing the particle density and by supposing a spherical shape, the sizes typify diameter of an equivalent sphere [23]. Therefore by SdFFF the particles are considered as compact spheres of uniform density, thus SdFFF to some extent hinders the mass of anisometric particles in spheres, resulting in this way in smaller dimensional values with respect to PCS. Notably, SdFFF is able to obtain higher resolution with respect to other light scattering methods since by SdFFF different particle fractions are firstly separated in different fractions and then sized, for this reason the technique is more accurate, particularly for those nanoparticles having broad and multimodal size distributions [23].

On the other hand, since PCS weighs particle dimension by light scattering, assuming a spherical shape, it can give misleading results in the case of non-spherical particles. Indeed the diffusion coefficient of anisometric particles is larger with respect to spheres of the same volume, so anisometric particles display a larger hydrodynamic radius by PCS with respect to SdFFF. Furthermore by PCS and in general by 
light scattering technique large particles monopolize the scattering behaviour of the sample [20]. Therefore by PCS the contribute of even a small number of large nanoparticles results in an appreciable enlargement of both size distribution and particle mean diameter with respect to SdFFF (Figure 3).

Finally, cryo-TEM can be considered as a local technique, where not all features of the samples may be imaged or artificially arranged (bigger objects may be excluded, other objects may be artificially arranged by size, orientation etc.). Therefore the use of cryo-TEM for measuring size could give ambiguous results because distances or geometries may be over- or underestimated [17].

Nevertheless cryo-TEM can be successfully employed for visualizing nanoparticles in an almost natural state, allowing to perform morphological assessment [31].

Figure 4 shows cryo-TEM images of SLN, SLN-P80 (panels A and B), NLC, NLC-P80 (panels C and D), fluorescent SLN-CG and SLNP80-CG (panels E and F).

Since lipids tend to crystallize in non-spherical platelet form, nanoparticles appear as ovoidal and ellipsoidal disk-like structures or as dark "cigars" when edge-on viewed [32]. Notably, the presence of aggregates is not detectable. In some cases a disk on top of another bigger one can be seen, showing a step or even several steps. Some smaller nanoparticles are a little bit tilted, allowing to clearly discern their ellipsoidal shape. The large particles that are positioned flat in the thickness of the frozen film (typically $200 \mathrm{~nm}$ thick) have much lower contrast with a small dark rim. Cryo-TEM analysis also confirmed that nanoparticle size and morphology were not affected by the lipid composition, P80 treatment and dye presence.

\section{Stability studies}

Stability studies were conducted in order to detect possible variations of morphology and size during nanoparticle storage, it is
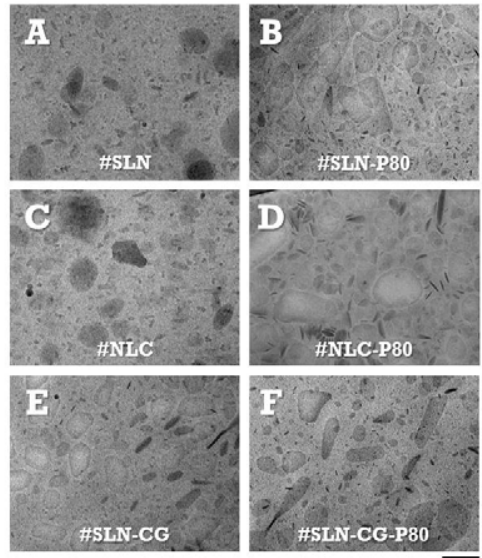

Figure 4: Effect of lipid nanoparticle constituents, presence of fluorescent dye and production parameters on particle dimensions and morphology, as determined by cryo-TEM analysis.

Pictures show a representative microscopic field of lipid nanoparticles constituted of solid lipids (A, B), a mixture of solid and liquid lipids (C, D) or solid lipids plus the fluorescent dye cardiogreen $(E, F)$.

After production, lipid nanoparticles were incubated at $40^{\circ} \mathrm{C}$, for $30 \mathrm{~min}$, in the absence $(A, C, E)$ or in the presence of polysorbate $80(B, D, F)$. For nanoparticle identification codes, composition and preparation procedure, please refer to Table 1 and experimental section. Bar corresponds to $200 \mathrm{~nm}$.
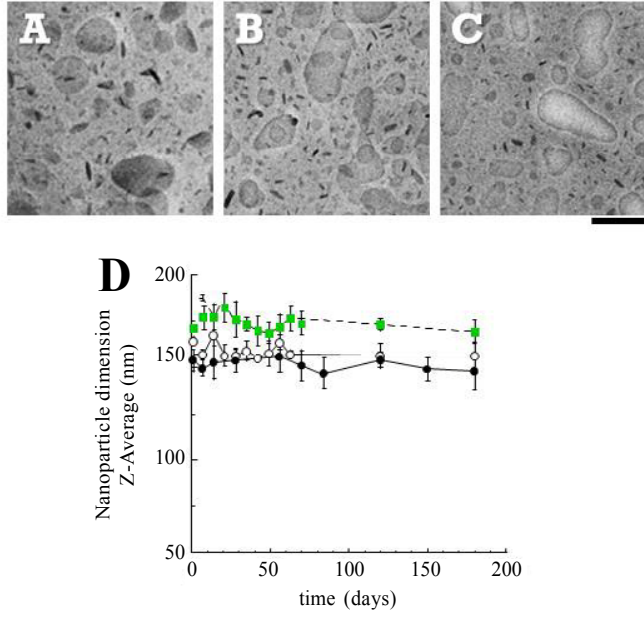

Figure 5: Effect of aging on the morphological (A-C) and dimensional (D) characteristics of lipid nanoparticles. Cryo-TEM photomicrographs were taken after 90 days from the preparation of \#SLN(A), \#SLN-P80 (B) and \#SLN-P80-CG $(C)$. Panel $D$ shows the variation of $Z$-average $( \pm S D, n=5)$ within the indicated length of time for \#SLN (open circles), \#SLN-P80 (filled circles) and \#SLNP80-CG (filled squares), data are the mean of 3 determinations on different nanoparticle batches. For nanoparticle identification codes, composition and preparation procedure, please refer to Table 1 and experimental section.

indeed known that the evaluation of stability is essential to assess the actual potential of colloidal carriers as drug delivery systems.

In this respect Figure 5 shows the effect of aging on morphology and Z-average mean diameters of SLN, SLN-P80 and SLN-P80-CG up to 6 months from production. As clearly displayed, nanoparticles retained their morphology, in addition all nanoparticle formulations resulted very stable in term of dimensions; mean diameters underwent indeed small variations along the entire period of analysis (i.e. up to 180 days). These data has confirmed previously reported studies from our laboratory $[17,33]$.

\section{In vitro uptake experiments}

In order to determine the ability of nanoparticles to be taken up by $\mathrm{BBB}$ cells, we have employed an in vitro model of human brain capillary endothelium based on the use of immortalized human brain endothelial cell line hCMEC/D3.

For these experiments, nanoparticles were fluorescently labelled with rhodamine and stability was tested in culture medium by determining the linearity between nanoparticle concentration and their fluorescence. Figure 6 reports the mean fluorescence values obtained after in vitro uptake experiments conducted on hCMEC/ D3 cells, employing fluorescent SLN-RH and SLN-P80-RH (panel A), NLC-RH and NLC-P80-RH (panel B). The analysis of the cellular uptake indicates that SLN and NLC were almost identically uptaken by the endothelial cells. This result suggests that the presence of lipid liquid constituent in the nanoparticle matrix did not greatly influence cell uptake, at least on the cellular model employed in our experiments.

Concerning P80 effect, both SLN-P80-RH and NLC-P80-RH displayed lower uptake values with respect to the corresponding control nanoparticles. This datum was tentatively attributed to the effect of the hydrophilic shell surrounding the P80 treated nanoparticles. The presence of polyoxyethylene chains responsible for in vivo avoidance of RES uptake appeared to negatively influence in vitro performances 

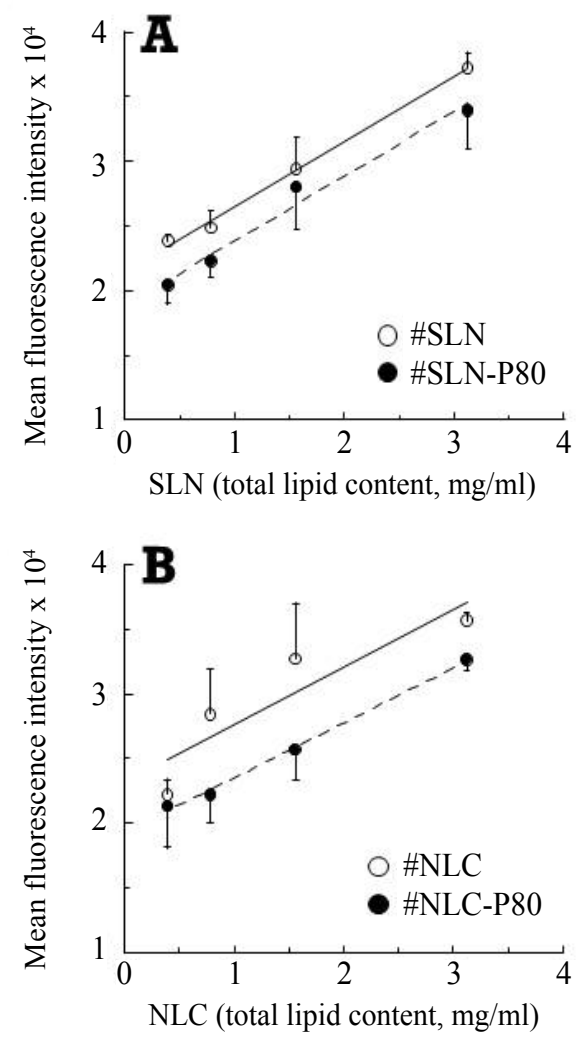

Figure 6: Effect of lipid nanoparticle constituents and production parameters on the uptake by human brain endothelial hCMEC/D3 cells. Data represent the mean \pm s.d. $(n=3)$ of the concentration-dependent experiments at $37^{\circ} \mathrm{C}$. Graphs show the dose response curve obtained treating the cells with the indicated dose of lipid nanoparticles constituted of solid lipids (A) or a mixture of solid and liquid lipids (B).

Data refer to lipid nanoparticles previously incubated at $40^{\circ} \mathrm{C}$, for $30 \mathrm{~min}$, in the absence (open circles) or in the presence of polysorbate 80 (closed circles). For nanoparticle identification codes, composition and preparation procedure, please refer to Table 1 and experimental section.

of nanoparticles. Probably the polyoxyethylene chains reduced the interactions between nanoparticle lipids and lipophilic membrane constituents, therefore reducing some of the possible internalization pathways postulated for the cell uptake of lipid nanoparticles, such as the formation of caveolae or lipid rafts [34].

\section{Biodistribution studies of SLN-P80-CG in mice}

In order to assess the in vivo biodistribution of nanoparticles, athymic mice experiments were performed after i.p. administration. Athymic nude mice were chosen to allow the detection of faint light signals coming from deepest anatomical districts, thanks to the absence of fur and hear bulbs, which are high absorbers and sources of light scattering. Animals were treated with SLN-P80-CG as well as by SLN-P80 administered as control. Notably, since we decided to use an exiguous number of animals for ethical and cost reasons, we performed only a preliminary study, employing SLN-P80-CG on the bases of the following considerations. P80 has a well-recognized stealth effect [35] and more importantly it has been suggested to possess a specific role in brain targeting $[28,29]$. For instance some studies have indicated that P80 inhibits the transporter P-glycoprotein, that prevents many endogenous and exogenous compounds crossing biological membranes [36]. Other studies have reported that nanoparticle coated with P80 could increase absorption of plasmatic apolypoprotein E on their surface, thus promoting brain passage through receptor mediated endocytosis [37,38].

The analysis of nanoparticle biodistribution was achieved by a preclinical research method based on non-invasive in vivo fluorescent luminescent imaging of small animals [22]. This technique can be considered as a systematic approach able to provide specific localization of nanoparticles, giving the possibility to image a whole animal. In addition, this technique allows performing further studies on specific isolated organs in which a signal was previously detected in vivo [21].

Figures 7 and 8 shows a selection of the acquired images, respectively depicting whole animals and isolated organs. Notably, all organs were imaged after complete perfusion, in order to eliminate traces of fluorescence from the vasculature. Particularly, photographs reported in Figures 7A to $7 \mathrm{H}$ refer to mice imaged in dorsal and ventral positions, respectively. In dorsal images fluorescent signals appear to be predominantly localized in the liver and spleen regions; in ventral images, as expected, the signal is diffusely localized in the whole peritoneal region, being the nanoparticles administered through the i.p. route. Notably, in one animal both in dorsal and ventral views, a fluorescent signal, even if of low intensity, is clearly detectable in a region corresponding to the brain.

As expected, no fluorescent signals are present in the images of animals treated by plain SLN-P80.

In order to investigate the distribution of nanoparticles in organs and to unambiguously localize the fluorescent dye accumulated in specific districts, different organs have been perfused and isolated.

As observable in Figure 8, showing the images of organs isolated from two representative animals, a predominant accumulation of

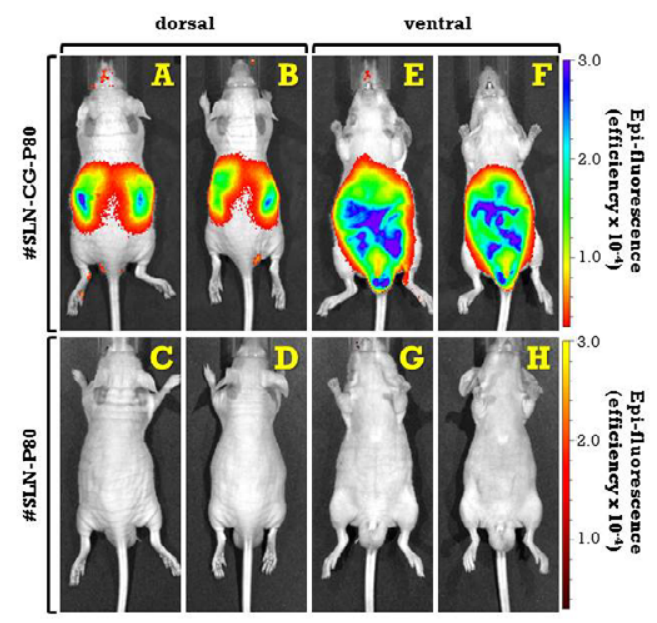

Figure 7: Optical images of athimic mice after intraperitoneal injection of 500 $\mu \mathrm{l}$ of \#SLN-CG-P80. Images were recorded after $4 \mathrm{~h}$ from the injection. In vivobody distribution of lipid nanoparticles, as determined by fluorescent luminescent imaging technology of total body.Pictures show the images collected from 2 representative animals that were treated, as described in the experimental section, with a dispersion of nanoparticles by intraperitoneal administration.

Dorsal $(A-D)$ and ventral $(E-H)$ images were recorded after the administration of fluorescent nanoparticles (\#SLN-CG-P80) (A, B, E, F) or blank, control nanoparticles (\#SLN-P80) (C, D, G, H). Colour bar on the right side indicates the signal efficiency of the fluorescence emission coming out from the animal. For nanoparticle identification codes, composition and preparation procedure, please refer to Table 1 and experimental section. 
Citation: Esposito E, Vries HED, Pol SMA, Boschi F, Calderan L, et al. (2015) Production, Physico-Chemical Characterization and Biodistribution Studies of Lipid Nanoparticles. J Nanomed Nanotechnol 6: 256. doi: 10.4172/2157-7439.1000256

Page 8 of 9

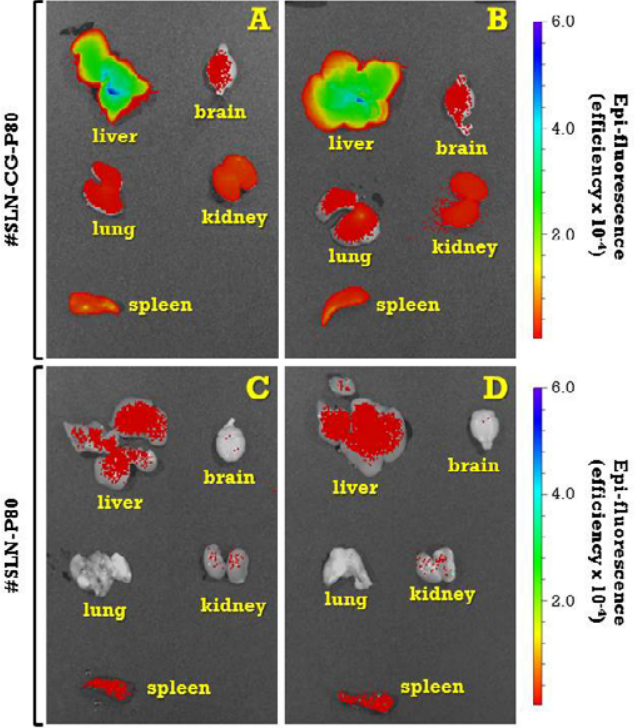

Figure 8: In vivo organ distribution of lipid nanoparticles, as determined by fluorescent luminescent imaging technology of isolated organs.Pictures show the images of organs isolated from 2 representative animals that were treated as described in the experimental section, with a dispersion of nanoparticles by intraperitoneal administration.

Organs are from mice treated with fluorescent nanoparticles (\#SLN-CG-P80) (A, B) or blank, control nanoparticles (\#SLN-P80) (C, D). Colour bar on the right side indicates the signal efficiency of the fluorescence emission coming out from the organ. For nanoparticle identification codes, composition and preparation procedure, please refer to Table 1 and experimental section.

nanoparticles occurred in the liver and in the spleen, typical store organs, nevertheless fluorescence was clearly detectable in other organs, including lung, kidney and notably in the brain [12].

This latest result is particularly interesting since it suggests that, notwithstanding the unavoidable tropism for RES district of lipid nanoparticles, the P80 functionalization enabled SLN-P80-CG to reach the brain in a detectable amount.

\section{Conclusions}

The reported results has evidenced that the complementary use of different techniques enables to fully characterize size, size distribution and morphology of nanoparticles, overcoming the specific drawbacks associated to any characterization technique. Notwithstanding the findings reported by other authors [8-10], in this study no large differences have been evidenced between NLC and SLN in terms of fluorescence loading and stability, at least for the composition of NLC and SLN tested in the current study. Therefore, this manuscript has demonstrated that NLC do not show any major advantage compared to SLN. The in vivo experiment proved that SLN-P80-CG were able to reach the brain, even in small proportion with respect to the injected dose, after i.p. administration. This finding has suggested that nanoparticle functionalization with polysorbate 80 could promote brain delivery, as described by other authors $[28,37,38]$.

Further studies will be undertaken to more specifically clarify if the presence of P80 could result in stealth effect. Poloxamer 188, employed to emulsify nanoparticle lipid matrix, can be also partially responsible for such effect. Indeed, as P80, also poloxamer 188 can modify the surface of SLN, causing a steric hindrance effect that would reduce RES uptake and prolong retention time in plasma.

\section{Acknowledgements}

This work was funded by "Futuro in Ricerca 2010 FIRB " from the Ministry of the University and Research of Italy.

\section{References}

1. Battaglia L, Gallarate M (2012) Lipid nanoparticles: state of the art, new preparation methods and challenges in drug delivery. Expert Opin Drug Deliv 9: 497-508.

2. Joshi MD, Müller RH (2009) Lipid nanoparticles for parenteral delivery of actives. Eur J Pharm Biopharm 71: 161-172.

3. Uner M, Yener G (2007) Importance of solid lipid nanoparticles (SLN) in various administration routes and future perspectives. Int J Nanomedicine 2: 289-300.

4. Nyström AM, Fadeel B (2012) Safety assessment of nanomaterials: implications for nanomedicine. J Control Release 161: 403-408

5. Puri A, Loomis K, Smith B, Lee JH, Yavlovich A, et al. (2009) Lipid-based nanoparticles as pharmaceutical drug carriers: from concepts to clinic. Crit Rev Ther Drug Carrier Syst 26: 523-580.

6. Yang $H(2010)$ Nanoparticle-mediated brain-specific drug delivery, imaging and diagnosis. Pharm Res 27: 1759-1771.

7. Göppert TM, Müller RH (2005) Polysorbate-stabilized solid lipid nanoparticles as colloidal carriers for intravenous targeting of drugs to the brain: comparison of plasma protein adsorption patterns. J Drug Target 13: 179-187.

8. Saupe A, Wissing SA, Lenk A, Schmidt C, Müller RH (2005) Solid lipid nanoparticles (SLN) and nanostructured lipid carriers (NLC) -- structural investigations on two different carrier systems. Biomed Mater Eng 15: 393-402.

9. Yoon G, Park JW, Yoon IS (2013) Solid lipid nanoparticles (SLNs) and nanostructured lipid carriers (NLCs): recent advances in drug delivery. J Pharm Invest 43: 353-362.

10. Shidhaye SS, Vaidya R, Sutar S, Patwardhan A, Kadam VJ (2008) Solid lipid nanoparticles and nanostructured lipid carriers--innovative generations of solid lipid carriers. Curr Drug Deliv 5: 324-331.

11. Zhang W, Liu J, Li S, Chen M, Liu H (2008) Preparation and evaluation of stealth Tashinone IIA-loaded solid lipid nanoparticles: Influence of Poloxame 188 coating on phagocytic uptake. J Microencapsul 25: 203-209.

12. Hirsjärvi S, Sancey L, Dufort S, Belloche C, Vanpouille-Box C, et al. (2013) Effect of particle size on the biodistribution of lipid nanocapsules: comparison between nuclear and fluorescence imaging and counting. Int J Pharm 453: 594 600

13. Esposito E, Mariani P, Ravani L, Contado C, Volta M, et al. (2012) Nanoparticulate lipid dispersions for bromocriptine delivery: characterization and in vivo study. Eur J Pharm Biopharm 80: 306-314.

14. Zahr AS, Davis CA, Pishko MV (2006) Macrophage uptake of core-shell nanoparticles surface modified with poly(ethylene glycol). Langmuir 22: 81788185

15. Kreuter J, Petrov VE, Kharkevich DA, Alyautdin RN (1997) Influence of the type of surfactant on the analgesic effects induced by the peptide dalargin after its delivery across the blood -brain barrier using surfactant-coated nanoparticles. J Control Rel 49: 81-87.

16. Ramge P, Unger RE, Oltrogge B, Zenker D, Begley D, et al. (2000) Polysorbate-80 coating enhances uptake of polybutylcyanoacrylate (PBCA) nanoparticles by human and bovine primary brain capillary endothelial cells. Eur J Neurosci 12: 1931-1940.

17. Esposito E, Fantin M, Marti M, Drechsler M, Paccamiccio L, et al. (2008) Solid lipid nanoparticles as delivery systems for bromocriptine. Pharm Res 25: 1521 1530.

18. Severino P, Pinho SC, Souto EB, Santana MHA (2011) Polymorphism, crystallinity and hydrophilic-lipophilic balance of stearic acid and stearic acidcapric/caprylic triglyceride matrices for production of stable nanoparticles. Colloids and Surfaces B, Biointerfaces 86: 125-130.

19. Jores K, Mehnert W, Drechsler M, Bunjes H, Johann C, et al. (2004) Investigations on the structure of solid lipid nanoparticles (SLN) and oilloaded solid lipid nanoparticles by photon correlation spectroscopy, field-flow fractionation and transmission electron microscopy. J Control Rel 95: 217-227. 
Citation: Esposito E, Vries HED, Pol SMA, Boschi F, Calderan L, et al. (2015) Production, Physico-Chemical Characterization and Biodistribution Studies of Lipid Nanoparticles. J Nanomed Nanotechnol 6: 256. doi: 10.4172/2157-7439.1000256

20. Egelhaaf SU, Wehrli E, Muller M, Adrian M, Schurtenberger P (1996) Determination of the size distribution of lecithin liposomes: a comparative study using freeze fracture, cryoelectron microscopy and dynamic light scattering. $J$ Microsc 184: 214-228.

21. Zelmer A, Ward TH (2013) Noninvasive fluorescence imaging of small animals. J Microsc 252: 8-15.

22. Boschi F, Spinelli AE, D'Ambrosio D, Calderan L, Marengo M, et al. (2009) Combined optical and single photon emission imaging: preliminary results. Phys Med Biol 54: L57-62.

23. Contado C, Blo G, Fagioli F, Dondi F, Beckett R (1997) Characterisation of River Po particles by sedimentation field-flow fractionation coupled to GFAAS and ICP-MS. Colloids and Surfaces, A: Physicochemical and Engineering Aspects 120: 47-59.

24. Pecora R (2000) Dynamic Light Scattering Measurement of Nanometer Particles in Liquids. J Nanoparticle Res 2: 123-131.

25. Weksler BB, Subileau EA, Perrière N, Charneau P, Holloway K, et al. (2005) Blood-brain barrier-specific properties of a human adult brain endothelial cell line. FASEB J 19: 1872-1874.

26. Kooij G, Kopplin K, Blasig R, Stuiver M, Koning N, et al. (2014) Disturbed function of the blood-cerebrospinal fluid barrier aggravates neuro-inflammation. Acta Neuropathol 128: 267-277.

27. Esposito E, Ravani L, Drechsler M, Mariani P, Contado C, et al. (2015) Cannabinoid antagonist in nanostructured lipid carriers (NLC): design, characterization and in vivo study. Mat Sci Eng C 48: 328-336.

28. Gulyaev AE, Gelperina SE, Skidan IN, Antropov AS, Kivman GY, et al. (1999) Significant transport of doxorubicin into the brain with polysorbate 80 -coated nanoparticles. Pharm Res 16: 1564-1569.
29. Tian XH, Lin XN, Wei F, Feng W, Huang ZC, et al. (2011) Enhanced brain targeting of temozolomide in polysorbate- 80 coated polybutylcyanoacrylate nanoparticles. Int J Nanomedicine 6: 445-452.

\section{Food and Drug Administration}

31. Tamjidi F, Shahedi M, Varshosaz J, Nasirpour A (2013) Nanostructured lipid carriers (NLC): A potential delivery system for bioactive food molecules. Innovative Food Science and Emerging Technologies 19: 29-43.

32. Sato K (1988) Crystallization and polymorphism of fats and fatty acids.

33. Esposito E, Ravani L, Contado C, Costenaro A, Drechsler M, et al. (2013) Clotrimazole nanoparticle gel for mucosal administration. Mater Sci Eng C Mater Biol Appl 33: 411-418.

34. Martins S, Costa-Lima S, Carneiro T, Cordeiro-da-Silva A, Souto EB, et al. (2012) Solid lipid nanoparticles as intracellular drug transporters: an investigation of the uptake mechanism and pathway. Int J Pharm 430: 216-227.

35. Ambruosi A, Yamamoto H, Kreuter J (2005) Body distribution of polysorbate-80 and doxorubicin-loaded [14C]poly(butyl cyanoacrylate) nanoparticles after i.v. administration in rats. J Drug Target 13: 535-542.

36. Amin ML (2013) P-glycoprotein Inhibition for Optimal Drug Delivery. Drug Target Insights 7: 27-34.

37. Wagner S, Zensi A, Wien SL, Tschickardt SE, Maier W, et al. (2012) Uptake mechanism of ApoE-modified nanoparticles on brain capillary endothelial cells as a blood-brain barrier model. PLoS One 7: e32568.

38. Kreuter J, Shamenkov D, Petrov V, Ramge P, Cychutek K, et al. (2002) Apolipoprotein-mediated transport of nanoparticle-bound drugs across the blood-brain barrier. J Drug Target 10: 317-325. 